\title{
Morphology and Interaction of Galaxies using Deep Learning
}

\author{
Fernando Caro ${ }^{1}$, Marc Huertas-Company ${ }^{1}$ and Guillermo Cabrera ${ }^{2,3}$ \\ ${ }^{1}$ GEPI, Observatoire de Paris, PSL Research University, CNRS, \\ Université Paris Diderot, Sorbonne Paris Cité, \\ 61 Avenue de l'Observatoire, 75014 Paris, France \\ email: fernando.caro@obspm.fr \\ ${ }^{2}$ Millenium Institute of Astrophysics, \\ Santiago, Chile \\ ${ }^{3}$ Center for Mathematical Modeling, Universidad de Chile, \\ Beaucheff 851, Santiago, Chile
}

\begin{abstract}
In order to understand how galaxies form and evolve, the measurement of the parameters related to their morphologies and also to the way they interact is one of the most relevant requirements. Due to the huge amount of data that is generated by surveys, the morphological and interaction analysis of galaxies can no longer rely on visual inspection. For dealing with such issue, new approaches based on machine learning techniques have been proposed in the last years with the aim of automating the classification process. We tested Deep Learning using images of galaxies obtained from CANDELS to study the accuracy achieved by this tool considering two different frameworks. In the first, galaxies were classified in terms of their shapes considering five morphological categories, while in the second, the way in which galaxies interact was employed for defining other five categories. The results achieved in both cases are compared and discussed.
\end{abstract}

Keywords. methods: data analysis, galaxies: structure, galaxies: interactions

\section{Introduction}

The morphological analysis of galaxies represents one of the main tools available for understanding the physical processes behind the formation and evolution of galaxies. However, thanks to surveys such as the Sloan Digital Sky Survey (SDSS), the availability of astronomical data has grown substantially in the last years, implying that morphological classification can not be performed anymore via visual inspection, which has been the approach traditionally employed for performing this task. The most successful solutions considered so far to deal with this problem rely, usually, on the utilization of machine learning for automating the classification task, being deep-learning one of the most promising options. By using deep-learning, Dieleman et al. (2015) were recently able of recover the classification of galaxies made by Galaxy Zoo with a near-perfect accuracy (more than $99 \%$ for galaxies that were classified with a high degree of agreement). Later, deep-learning was successfully used by Huertas-Company et al. (2015) for also classifying galaxies at high-redshift, which represents a major improvement respect to Concentration-Asymmetry-Smoothness (CAS) based methods since the fraction of missclassifications is specially relevant at high-redshift (20\%-30\%).

While in terms of accuracy deep-learning offers a significat improvement in comparison with the methods previously used, its utilization for different problems (or even for the same problem but with different data) should take into account several details that can have a big influence over the results. For example, the architecture of the convolutional 

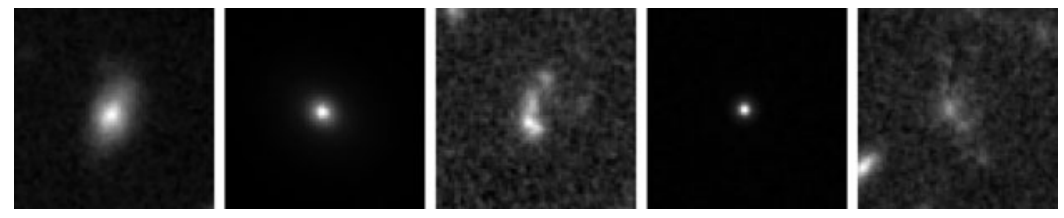

Figure 1. Five morphological categories, from left to right: disk, spheroid, peculiar/irregular, point source/compact and unclassifiable.
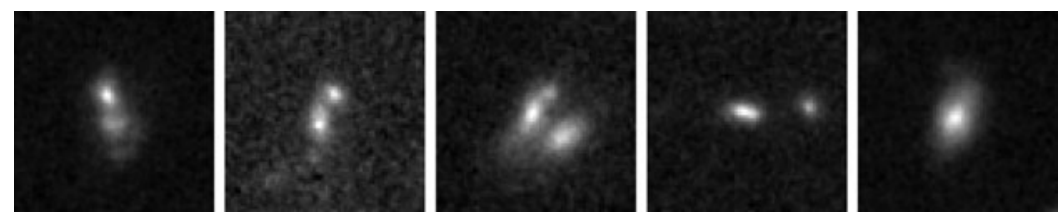

Figure 2. Five interaction categories, from left to right: merger, interaction within segmentation-map, interaction beyond segmentation-map, non-interactive companion and none.

neural network and the specific characteristics of the training set can dramatically affect the predictive capabilities of deep-learning.

Another problem, that is closely related the to morphological one, consists in the detection and classification of interacting galaxies. This second problem can be also considered as a morphological one, but where the categories are defined by more complex shapes for the galaxies and then described by more parameters. Therefore, considering all that was mentioned before, our aim is to explore the feasibility of using the same deep-learning architecture employed in the morphological problem for the case where galaxies should be classified according to their interaction patterns.

\section{Convolutional neural network architecture and data employed}

A simpler version of the convolutional neural network's architecture used for morphological classification was employed for our experiment. Our network consists in three convolutional layers (including two max-pooling steps after the first and the third layer) and one fully conected layer. In the case of the network employed for the morphological problem, five convolutional layers are followed by two fully conected ones.

In order to test the performance of the network in the morphological and also in the interaction classification problem, for then being able of comparing both results, we had used the catalogue released by Kartaltepe et al. (2015) for the GOODS-S field of CANDELS (Grogin et al. 2011, Koekemoer et al. 2011). In this catalogue, 7634 galaxies, observed in 5 different bands, are classified in terms of their respective morphologies (main morphology class) and their interaction patterns (interaction class), among many other descriptors included in the CANDELS scheme. In both cases, five categories are considered. The five morphological categories correspond to disk, spheroid, peculiar/irregular, point source/compact and unclassifiable (Fig. 1), while the five interaction categories are given by merger, interaction within segmentation-map, interaction beyond segmentationmap, non-interactive companion and none (Fig. 2).

From the data available for GOODS-S, we employed images of two bands (F125 and F160), which means that we had around 15,000 images of galaxies with their corresponding morphological and interaction probabilities for training and testing the network. Two thirds of the total amount of images (around 10,000 images) were employed for the training the network, while the remaining third (around 5,000 images) was used for studiying 
Table 1. Results obtained in the two classification problems and the corresponding weights of the five categories considered in each problem.

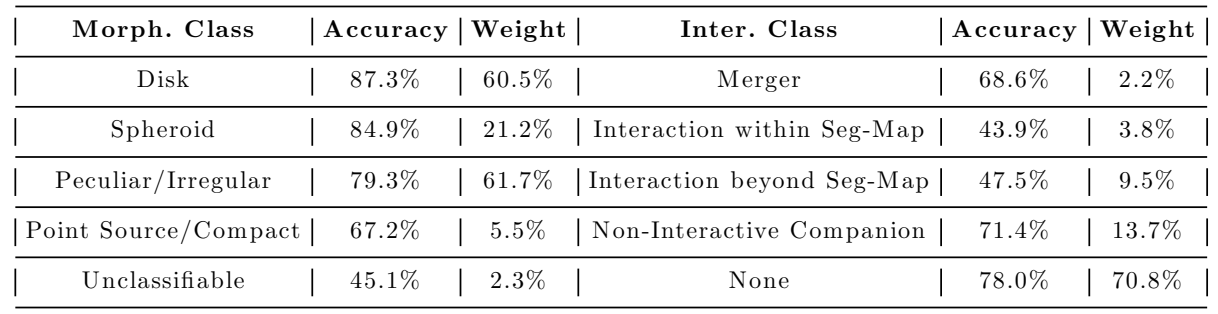

the accuracy achieved by the network in these two classification problems. All the other parameters requiered by the network were kept ideantical for both problems.

\section{Performance results}

The results obtained for the classification of the images in the two problems are specified in Table 1. The accuracy achieved by the network for each one of the five classes is defined as the percentage of images corresponding to such class that are correctly classified after the training stage. The accuracy results are presented with the corresponing weight of each one of the classes considering all the images used during the training, expressed also as percentage. In the left side of the table we have the results obtained for the morphological problem, while in the right side we have the results obtained for the interaction problem.

For the morphological problem, the results suggest that the accuracy achieved by the network is proportional to the weight of the corresponding class, while, in the case of the interaction problem, this relation is not valid. In fact, for the interaction problem, we can notice that the category with the smallest weight (merger) in the training set, does not have the worst accuracy results. It should be also pointed out that the two classes of interaction within and beyond the segmenetation map have similar accuracy results, regardless the fact the weight of the second one is 2.5 times the weight of the first one. This strongly suggests that the network is very inefficient in distinguish an image between this two categories. On the other hand, the limitations of the machine with this two categories can be the reason for the high accuracy achieved in the case of the merger class, despite having the smallest weight in the training set, since the confussion between two categories can yield to distinguish a third category in an easier way.

\section{Concluding remarks}

The results previously presented indicate that it is actually feasible to use the same architecture of a convolutional neural network employed to classify galaxies, according to their morphologies, for classifying them in terms of their interaction patterns. However, the results obtained will not be optimal since two categories are confused by the network, which yields to an increase in the missclassifications of the galaxies. The most likely reason behind this is that the network's architecture is not the best for detecting the features required to clearly dinstinguish the five interaction classes. In that sense, using this network is a good starting point, but it should be modify in order to perform in an optimal way in the interaction problem.

It is also important to highlight the strong influence of the weight of the categories in the training set. The results obtained seem to be absolutely affected by having a class 
that is too big in comparison with the rest, as it happens with the disk category, that represents more than the $60 \%$ of the training set of the morphological problem, and with the none category in the case of the interaction problem, where it represents more than the $70 \%$. Therefore, it would be interesting to repeat the experiments using a synthetic training set, with all the categories balanced in terms of weight, to analyze in this way if the results can change drastically due to different weight distributions.

Finally, the size of the training set can have also affected the accuracy achieved by the network, since it is well known that deep-learning is very sensible to the size of the training set. Then, it would be also interesting to repeat the experiments using a bigger training to verify if there is a change in the results obtained. Nonetheless, usually in many astronomical problems there is no chance of getting more data. Probably, the only option to deal with a size problem in the training set in a case like that is to use simulations where the trends observed in practice are replicated, otherwise many biases would be introduced during the training of the network leading to new missclassification problems. Therefore, for the specific case of galaxy morphology and interaction, the usage of hydrodynamical cosmological simulations, such as Illustris (Genel et al. 2014) or Horizon-AGN (Dubois et al. 2016), can be useful to build realistic training sets and that are also big enough to allow an optimal training of any convolutional neural network.

\section{References}

Dubois, Y., Peirani, S., Pichon, C., et al. 2016, MNRAS, 463, 3948

Genel, S., Vogelsberger, M., Springel, V., et al. 2014, MNRAS, 445, 175

Kartaltepe, J. S., Mozena, M., Kocevski, D., et al. 2015, ApJ (Series), 221, 11

Huertas-Company, M., Gravet, R., Cabrera-Vives, G., et al. 2015, ApJ (Series), 221, 8

Dieleman, S., Willett, K. W., \& Dambre, J. 2015, MNRAS, 450, 1441

Koekemoer, A. M., Faber, S. M., Ferguson, H. C., et al. 2011, ApJ (Series), 197, 36

Grogin, N. A., Kocevski, D. D., Faber, S. M., et al. 2011, ApJ (Series), 197, 35 